\title{
Burden of heart diseases in children attending cardiology clinic in a regional referral hospital in Uganda
}

\begin{abstract}
Introduction: Patterns and prevalence of heart diseases are different between the high income countries and low income countries. Acquired heart diseases like RHD, cardiomyopathies, pericarditis are still a public health burden while congenital heart diseases still carry a poor outcome among children. The aim of this study was to report the burden and pattern of heart diseases in a semi urban referral hospital in Uganda.
\end{abstract}

Methods: This was a prospective study in the paediatrics department at a regional referral hospital in south western Uganda over a period of 20 months. All children from birth up to 12 years who were referred or presented at the paediatrics department from different areas in the region for an index cardiac evaluation and had their transthoraxic echo done were included in the study. These children were enrolled and followed up in the cardiology clinic for chronic care. Those who did not turn up for their expected reviews were followed up with phone calls to find out their outcome.

Results: Two hundred and forty children were screened during this period and $158(65.8 \%)$ had abnormal echocardiological findings. More females than males were had abnormal Echos at $51.9 \%$. VSD were the commonest acyanotic heart disease followed by PDA at $37 \%$ and $17 \%$ respectively. TOF has the highest prevalence at $8.23 \%$ followed by Truncus arteriosus at $5.9 \%$ amongst the cyanotic heart diseases. Mean age at diagnosis of congenital heart disease was 28months (SD 33). RHD was the commonest acquired heart diseases $(72.5 \%$.) Sixty three percent of children needed and were referred to the national cardiac centre. Only $52 \%$ of referred children managed to go to the national referral cardiac centre. Of all the children who needed surgical intervention, $4.4 \%$ got the intervention. $25 \%$ of the children died while $12.7 \%$ were lost to follow up.

Conclusion; There is a high burden of heart diseases in south western Uganda with limited access to interventional services.
Volume 4 Issue 4 - 2017

\author{
Dorah Nampijja,' Elias Kumbakumba,' \\ Francis Bajunirwe,' Charles Mondo² \\ 'Mbarara University of Science and Technology, Uganda \\ ${ }^{2}$ Mulago National Referral Hospital, Uganda
}

Correspondence: Dorah Nampijja, Mbarara University of Science and Technology, P.O. Box 1410, Mbarara, Uganda, Email drdorah@yahoo.com

Received: March 12, 2017 | Published: May 09, 2017

\section{Background}

Patterns and prevalence of heart diseases are different between the high income countries and low income countries. ${ }^{1,2}$ Acquired heart diseases like RHD, cardiomyopathies, pericarditis are still a public health burden while congenital heart diseases still carry a poor outcome among children. ${ }^{3}$ Uganda is one of the countries that contribute to the highest prevalence of congenital heart disease in Sub Saharan Africa. ${ }^{4}$ The absence of early diagnosis, surgical intervention and inadequate access to medication coupled with the existing burden of communicable diseases increases the strain on a staggering economy of most sub Saharan countries. ${ }^{4}$ Unlike in the developed countries, children with heart disease in LMIC present late with severe complications to hospitals that are inadequately equipped to manage both the simple and complex heart diseases..$^{5-8}$ Over the years, good will organizations have come to aid in providing surgical intervention for the children with heart diseases within and out of their countries. ${ }^{2-8}$ However, the burden still remains unclear and the outcome of the children who don't get the opportunity of surgery is still not well known.

There is limited awareness of the burden of heart disease among children in SSA, Uganda inclusive. The aim of this study was to report the prevalence and pattern of heart diseases in a semi urban referral hospital in Uganda. Knowing how big the problem of Heart disease is in Uganda would be a right step in planning and future management of heart diseases in children. Furthermore the study would help create insight into the economic and social burden placed on the health system and patients' families.

\section{Methods}

This study was conducted in a regional referral hospital in south western Uganda. This is a predominantly rural and poor region with recent limited access to diagnosis, no access to corrective procedures and constrained management for cardiac diseases in children this was a prospective study in the paediatrics department at a regional referral hospital in Uganda over a period of 20months. All children from birth up to 12 years who were referred to or presented at the paediatrics department from different areas in the region for an index cardiac evaluation and had their transthoraxic echo done were included in the study. Age, sex, indication for echocardiography and cardiac diagnosis of every patient with an abnormal echocardiogram presenting to the clinic were recorded. Trans-thoracic two dimensional guided M-mode echo-cardiography using commercially available echocardiography machine (Siemens Colour Diagnostic Ultra sound System Model Acuson P300). We performed Echocardiographic examination in the sub costal, suprasternal views parasternal long axis, short axis 
and apical four chambers. Parameters recorded included presence and identification of structural heart defects, left ventricle diameter in diastole (LVIDD) and left Atrium diameter(LAD), systolic dysfunction (ejection Fraction $<45 \%$ ).

Valvular heart diseases were diagnosed based on the following findings:

i. Mitral stenosis: presence of thickened and calcified mitral valve leaflets and the abnormal shape of the anterior mitral valve leaflet (Hockey stick deformity) and the mitral valve area of less than $1.5 \mathrm{~cm}^{2}$ by planimetry. ${ }^{9}$

ii. Mitral Regurgitation: poor coaptation of the mitral valve leaflets in systole, thickened leaflets, dilated and hyper dynamic left ventricle, prolapsed anterior mitral valve leaflets and presence of turbulence on colour Doppler at the mitral valve.

iii. Aortic stenosis:presence of calcified and thickened aortic valves with poor separation

iv. Aortic regurgitation: poor coaptation of the aortic cusps in diastole, dilated left ventricles and turbulence on colour Doppler Rheumatic Heart disease (RHD) was defined by the presence of any definite evidence of mitral or aortic valve regurgitation seen in two planes by the TTE with the following morphologic abnormalities of the regurgitating valves: restricted leaflet mobility or focal or generalized valvular thickening, or abnormal subvalvular thickening. Dilated cardiomyopathy was diagnosed if there were dilated heart chambers with impaired LV systolic function. ${ }^{10}$ Pericardial effusion was diagnosed when there was echo free space between the visceral and parietal pericardium. ${ }^{11}$

These children were enrolled and followed up in the cardiology clinic for chronic care. Those who did not turn up for their expected reviews were followed up with phone calls to find out where they were at and what was happening to them. We documented any other outcomes like honouring referral, surgical intervention, loss to follow up in the cardiology clinic and death. Ethical approvals were obtained from the institutional review board

\section{Results}

Two hundred and forty children were screened during the period and $158(65.8 \%)$ of these children had abnormal echocardiolographical findings. More females than males were had abnormal echo findings at $51.9 \%$. Mean age at diagnosis of congenital heart disease was 28 months (SD 33). Presence of a murmur at $41 \%$ was the most frequent indication for performing an Echo was followed by other symptoms like dysmorphism and cyanosis (Figure 1). Acyanotic heart lesions were predominant at $55 \%$ while $25 \%$ vof all echo abnormalities were due to acquired heart diseases. Ventricular septal defects were the commonest acyanotic heart disease followed by PDA at $37 \%$ and $17 \%$ respectively. Tetralogy of Fallot was the commonest cyanotic heart disease at $8.23 \%$ followed by Truncus arteriosus at $5.9 \%$ (Table 1 ).

Rheumatic heart disease (RHD) was the commonest acquired heart disease at $72.5 \%$ of the acquired heart diseases and majority had mitral regurgitation (96.5\%). Majority of children with RHD (45\%) had associated dilated left heart chambers. Other acquired lesions were pericardial effusion, pericarditis and dilated cardiomyopathy. Of all patients with abnormal echo findings, 131(63\%) needed surgical intervention and were referred to the national cardiac centre. Only $52 \%$ managed to go to the cardiac centre. Of all children with heart diseases that needed intervention, only $4.4 \%$ got surgical intervention (at 3PDA closure, 2VSD repair and 1valve replacement). At the time of analysis, $25 \%$ of the children had died while $12.7 \%$ were lost to follow up of all the children who died, $72.5 \%$ did not turn up at the referral centre.

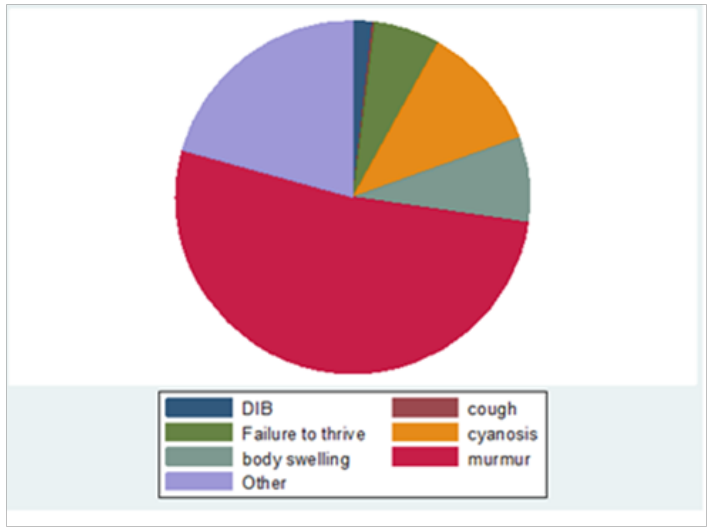

Figure I A pie chart showing indication for echocardiography.

Table I showing distribution of heart disease by sex

\begin{tabular}{|c|c|c|c|c|}
\hline \multirow[b]{2}{*}{ Type of lesion } & \multicolumn{2}{|l|}{ Sex } & \multirow[b]{2}{*}{ Total } & \multirow[b]{2}{*}{ Percentage } \\
\hline & Female & Male & & \\
\hline RHD & 20 & 20 & 40 & 25 \\
\hline Other & 2 & 0 & 2 & \\
\hline VSD & 27 & 17 & 44 & 27.85 \\
\hline ASD & 5 & 5 & 10 & 6.33 \\
\hline PDA & 9 & 11 & 20 & 12.66 \\
\hline $\begin{array}{l}\text { Complete AV Canal } \\
\text { Defect }\end{array}$ & 6 & 2 & 8 & 5.06 \\
\hline Pulmonary Stenosis & 2 & 2 & 4 & 2.5 \\
\hline TOF & 4 & 9 & 13 & 8.23 \\
\hline TGA & 1 & 1 & 2 & 1.27 \\
\hline Truncus Arteriosus & 2 & 5 & 7 & 4.43 \\
\hline Pulmonary Atresia & 1 & 0 & & 0.63 \\
\hline Complex heart diseases & 3 & 4 & 10 & 4.43 \\
\hline Total & 82 & 76 & 158 & 100 \\
\hline
\end{tabular}

\section{Discussion}

Heart diseases still cause significant morbidity \& mortality in children. Our study aimed to profile the different heart diseases in children and compare with other centres worldwide. We hoped to estimate the burden and outcome of children with heart diseases seen at a regional referral centre in a low income country. There were 158patients with heart diseases with a female to male ratio of 1.1:1. This is different from studies that have looked at CHD only where more males were affected. In our study we looked at both the congenital and acquired heart diseases. Like in most other studies, among the congenital abnormalities, acyanotic heart diseases were the commonest at $55 \%$ with VSD as the predominant lesion similar to what has been found in other studies. We had a relatively high number of children with $\mathrm{PDA}(12.5 \%)$ which is different from what others studies have found. After VSD, the commonest heart lesion was ASD. This could be explained by the fact that ASDs rarely present with symptoms yet most patients we screened were referred in with symptoms suggestive of cardiac disease. We also found a considerable number of complete AV canal defects at $5.06 \%$. This is higher than the $3 \%{ }^{12}$ seen in the general population. This could be explained by the fact that ours was a small selected population 
Among the cyanotic congenital heart diseases, TOF was predominant at $8.23 \%$ of all the children with heart diseases. This is similar to most studies but lower than what has been observed in Asia ${ }^{13,14}$ probably due to increased consanguinity that is common in Asia compared to our population. This was followed by Truncus arteriosus and other complex heart lesions. We noticed that there was late presentation and diagnosis of congenital heart disease in our patient. This is similar to what happens in most LMICs and different from what is reported developed countries where diagnosis is done as early as the prenatal period. This is explained by the lack of awareness of heart disease among low level health workers and the lack of access to diagnostic amenities that result from poverty and poor socioeconomic status. Acquired heart diseases, constituted 25\% of all the heart diseases of the children in our study. The predominant cause was Valvular diseases secondary to RHD. Mitral regurgitation was the most frequent lesion among the valvular heart disease and there was associated left heart chamber dilatation in $44.8 \%$ of children. The predominance of MR was similar to studies done in Mozambique, Cambodia and at Mulago among RHD patients. ${ }^{15,16}$ There was a challenge of accessing referral services. Only $52 \%$ of 131 children referred arrived at the referral centre. Most patients come from poor families, subsistence cost and the cost of transport to the referral centre was so high for them to afford. Furthermore, the limited access to intervention was similar to other sub Saharan centres. By the end of the study period, 25\%(40) of the children diagnosed with heart disease had died. This is higher than what has been seen in developed countries at $4 \%$ and $8 \%$ in Belgium and USA respectively. ${ }^{17}$ This is probably due to the disparity in access to surgical intervention in the two worlds.

\section{Conclusion}

The burden of heart disease in children is high in south western Uganda; with limited access to interventional services. This has resulted into increased mortality amongst children with heart diseases.

\section{Acknowledgements}

Research reported in this publication was supported by the Fogarty International Center (FIC), Office of the Director National Institutes of Health (OD), National Institute of Mental Health (NIMH), National Institute of Neurological Disorders and Stroke (NINDS) of the National Institutes of Health under Award Number D43 TW010128. The content is solely the responsibility of the authors and does not necessarily represent the official views of the National Institutes of Health.

\section{Conflict of interest}

The author declares no conflict of interest.

\section{References}

1. van der Linde D, Konings EE, Slager MA, et al. Birth prevalence of congenital heart disease worldwide: a systematic review and meta-analysis. Journal of the American College of Cardiology. 2011;58(21):2241-2247.
2. Zühlke L, Mirabel M, Marijon E. Congenital heart disease and rheumatic heart disease in Africa: recent advances and current priorities. Heart. 2013;99(21):1554-1561.

3. Marijon E, Ou P, Celermajer DS, et al. Prevalence of rheumatic heart disease detected by echocardiographic screening. $N$ Engl J Med. 2007;357(5):470-476.

4. Edwin F, Entsua-Mensah K, Sereboe LA, et al. Conotruncal heart defect repair in Sub-Saharan Africa: remarkable outcomes despite poor access to treatment. World J Pediatr Congenit Heart Surg. 2016;7(5):592-599.

5. Hewitson J, Brink J, Zilla P. The challenge of pediatric cardiac services in the developing world. Seminars in Thoracic and Cardiovascular Surgery. 2002;14(4):340-345.

6. Mocumbi AO, Lameira E, Yaksh A, et al. Challenges on the management of congenital heart disease in developing countries. Int $J$ Cardiol. 2011;148(3):285-288.

7. Mehta A, Saxena A, Juneja R, et al. Characteristics and outcomes of Indian children enrolled in a rheumatic heart disease registry. Int $J$ Cardiol. 2016;222:1136-1140.

8. Dearani JA, Neirotti R, Kohnke EJ, et al. Improving pediatric cardiac surgical care in developing countries: matching resources to needs. Seminars in Thoracic and Cardiovascular Surgery: Pediatric Cardiac Surgery Annual. 2011;13(1):35-43.

9. Baumgartner H, Hung J, Bermejo J, et al. Echocardiographic assessment of valve stenosis: EAE/ASE recommendations for clinical practice. $J$ Am Soc Echocardiogr. 2009;22(5):442.

10. Bonow RO, Carabello B, de Leon AC, et al. Guidelines for the management of patients with valvular heart disease executive summary a report of the American college of cardiology/american heart association task force on practice guidelines (committee on management of patients with valvular heart disease). Circulation. 1998;98(18):1949-1984.

11. Feigenbaum H. Echocardiographic diagnosis of pericardial effusion. The American Journal of Cardiology. 1970;26(5):475-479.

12. Calabrò R, Limongelli G. Complete atrioventricular canal. Orphanet $J$ Rare Dis. 2006;5(1):8.

13. Amro K. Pattern of congenital heart disease in Jordan; 2010. 14 p.

1. Sharmin LS, Haque MA, Bari MI, et al. Pattern and clinical profile of congenital heart disease in a teaching hospital. TAJ: Journal of Teachers Association. 2008;21(1):58-62.

14. Marijon E, Ou P, Celermajer DS, et al. Prevalence of rheumatic heart disease detected by echocardiographic screening. $N$ Engl $J$ Med. 2007;357(5):470-476.

15. Lubega S, Aliku T, Lwabi P. Echocardiographic pattern and severity of valve dysfunction in children with rheumatic heart disease seen at Uganda heart institute, Mulago hospital. Afr Health Sci. 2014;14(3):617-625.

16. Bernier P-L, Stefanescu A, Samoukovic G, Tchervenkov CI. The challenge of congenital heart disease worldwide: epidemiologic and demographic facts. Seminars in Thoracic and Cardiovascular Surgery: Pediatric Cardiac Surgery Annual. 2010;13(1):26-34. 